\title{
CONF-9008179 -..1
}

\section{Automatic Evaluation of First - and Higher-Derivative Vectors ${ }^{1}$}

\author{
Andreas Griewank \\ Mathematics and Computer Science Division \\ Argonne National Laboratory \\ Argonne, IL 60439-4801, U.S.A.
}

\begin{abstract}
The numerical analysis of parameter-dependent nonlinear systems almost always involves first-derivative matrices and often also requires the evaluation of higher derivatives along certain singular directions. It is shown here how these selected derivative values can be obtained without differencing and at a reasonable cost under the realistic assumption that the nonlinear system is given in the form of a computer program for its evaluation.
\end{abstract}

\section{Introduction and Basic Assumptions}

Over the past two decades great advances have been made regarding the theoretical and numerical analysis of nonlinear systems

$$
f(x)=0 \quad \text { with } f: \mathrm{R}^{n} \rightarrow \mathrm{R}^{m} .
$$

Often such finite-dimensional systems belong to a family of discretizations for an underlying differential or integral equation. Apart from the implicit understanding that the number $n$ of independent variables and the number $m$ of dependent variables may be very large, we will not have to consider this discretization aspect explicitly. In particular, sparsity is tak an into account implicitly. Typically, the argument $x \in \mathrm{R}^{n}$ combines a state vector with various design and control parameters, plus possibly the time. We will not distinguish the role of these independent variables, because derivatives of the same kind and order are usually required with respect to all of them.

Usually the difference $m-n$ is a small positive integer equal to the largest codimension of any singularity in the region of interest. Sometimes the number $n$ may also be increased by introdicing imperfection parameters in order to make certain singularities generic. In such cases, it is best to label all potentially varying parameters as independent variables in the first place, even though some of them may be constant for most of the calculation. As we will see, the complexity estimate for the evaluation of the Jacobian matrix

$$
J(x)=f^{\prime}(x) \in \mathrm{R}^{m \times n}
$$

\footnotetext{
${ }^{1}$ This work was supported by the Applied Mathematical Sciences subprogram of the Office of Energy ReseatfeD U.S. Department of Energy, under Contract W-31-109-Eng-38. 
in the so-called reverse mode of automatic differentiation is in fact completely independent of the number $n$ of independent variables. In other words, there is essentially no computational penalty for considering many input parameters as variable. This fact may be quite helpful for a sensitivity analysis after solutions have been obtained with sufficient accuracy.

Especially if the finite-dimensional system is obtained by an adaptive discretization or involves piecewise smooth approximations, e.g., splines, the vector function $f$ may have cracks, kinks (discontinuities in $J(x)$ ), or jumps in higher derivatives. Indeed, in many applications, $f(x)$ will not even be globally defined at all $x \in \mathbf{R}^{n}$. To avoid any unnecessary complications, we will assume throughout that $f(x)$ is sufficiently often differentiable near all arguments of interest. The resulting computational techniques are likely to work as long as the underlying discretization converges in the appropriate Sobolev norm so that the discontinuities in all relevant derivatives become smaller and smaller as the grid is refined.

The chain-rule based technique of automatic differentiation has been used for more than 30 years, mostly in the so-called forward mode. For a recent survey, see [4]. We restrict our attention to derivative vectors, because a direct accumulation of derivative matrices or tensors involves more complicated data structures and raises combinatorial optimization problems [5].

\section{Required Derivatives}

Wherever some submatrix of the Jacobian $J(x)$ has full row rank $m$, the solution set $f^{-1}(0)$ forms locally a smooth $(m-n)$-dimensional manifold. The same is true for the slices obtained by fixing the values of variables whose partials are not contained in a particular nonsingular submatrix. Any of these manifolds may be locally parametrized in terms of a subset of the independent variables or some other local coordinates. In either case the corresponding local chart is an implicit function that is often approximately evaluated by using a few steps of Newton's method. Typically, Newton steps are calculated by first forming and then factoring the relevant square Jacobian, which we may assume to be a submatrix of $J(x)$. While this approach may be quite costly in general, the Jacobian often has a regular sparsity pattern or a priori known spectral properties that allow the computation of an approximate Newton step at a reasonable cost. Even when such structural information is not available, one may be able to obtain Newton steps comparatively cheaply by solving an extended system without forming the Jacobian at all. This approach was outlined in [5] and will not be pursued further here.

When a particular submatrix of the Jacobian is singular, the implicit function theorem no longer applies, and the structure of the corresponding slice depends on the size of the rank drop as well as the properties of the second-derivative tensor $\nabla^{2} f(x) \in \mathrm{R}^{m \times n \times n}$. Fortunately one does not need to evaluate the full tensor but merely its restriction to the null spaces of the relevant submatrix 
and its transpose, respectively. Let us assume for notational simplicity that at some point $x_{*}$ the latter is spanned by a single vector $0 \neq u \in \mathcal{R}^{n}$. Correspondingly, the null space of the submatrix itself is spanned by the columns $\left\{v_{i}\right\}_{i=0 \ldots m-n}$ of an $n \times(m-n+1)$ matrix $V$. Then the structure of the solution set for $x \approx x_{*}$ depends on the symmetric matrix

$$
H(x)=\left[u^{T} \nabla^{2} F(x) v_{i} v_{j}\right]_{j=0,1,1, \ldots m-n}^{i=0, \ldots m-n} .
$$

Provided $H\left(x_{*}\right)$ is nonsingular, it is well known that $x_{*}$ is an isola formation or a bifurcation point, depending on whether $H\left(x_{*}\right)$ is definite or not. Otherwise $x_{*}$ is a cusp singularity in that $H\left(x_{*}\right)$ has a nontrivial nullvector $c \in \mathcal{R}^{m-n+1}$, and one has to look into the next-higher derivative, namely, the cubic term

$$
\left.h(x) \equiv u^{T} \nabla^{3} f(x)(x) v^{3} \equiv \frac{\partial^{3}}{\partial t^{3}} u^{T} f(x+t v)\right|_{t=0},
$$

where $v=V c \in \mathbf{R}^{n}$. Similarly, higher singularities (see e.g. [10]) are characterized by the vanishing of one or more components or dot products of partial derivative vectors of the form

$$
b(x) \equiv \nabla^{d} f(x) v_{1} v_{2} \ldots v_{d}=\left.\frac{\partial^{d} f\left(x+t_{1} v_{1}+t_{2} v_{2}+\cdots+t_{d} v_{d}\right)}{\partial t_{1} \partial t_{2} \cdots \partial t_{d}}\right|_{t_{i}=0} .
$$

Moreover, to rapidly approximate bifurcation points, cusp singularities, or other higher order degeneracies by Newton's method, one also needs the gradients of the scalar quantities that vanish at the singularity in questions. As shown, for example, in [7] for the cusp case, these gradients are linear combinations of $n$-vectors of the form

$$
a(x) \equiv u^{T} \nabla^{d+1} f(x) v_{1} v_{2} \ldots v_{d}=\nabla_{x}\left[\frac{\partial^{d} u^{T} f\left(x+t_{1} v_{1}+t_{2} v_{2}+\cdots+t_{d} v_{d}\right)}{\partial t_{1} \partial t_{2} \cdots \partial t_{d}}\right]_{t_{i}=0} .
$$

Expressions of this form that are relevant at Hopf points, Takens-Bog(lanov singularities, and other bifurcations of dynamical systems are given in [1]. In both (1) and (2) the direction vectors $v_{i}$ may well be restricted to a (state) subspace; but, as we discussed above, the extra level of differentiation in (2) compared to (1) can be carried out with respect to any potentially varying parameter as well.

We will refer to the $n$-vector $a$ given in (2) as an adjoint of the $m$-vector $b$ defined in (1). Generally, an adjoint of an arbitrary vector function $b$ is obtained by forming the dot product of $b$ with some weight vector $u$ and then differentiating the resulting scalar with respect to the full vector of independent variables $x$. For $d=0$ and with $u$ ranging over all Cartesian basis vectors in $\mathbf{R}^{n}$, one may obtain from (2) the Jacobic $\mathrm{n} J(x)$ row by row. When $n$ is significantly larger than $m$, this scheme is likely to be more efficient than the alternative of picking up successive columns $b$ as defined in (1) with $d=1$ and $v_{1}$ ranging over the Cartesian basis vectors in $\mathrm{R}^{n}$. Howevor, this rule of thumb need not always apply, and there are mixed modes that yield the Jacobian even more cheaply, at least in certain cases.

Obviously the accuracy with which the direct and adjoint derivative vectors $b$ and $a$ as defined in (1) and (2) can be approximated by divided differences diminishes rapidly as the degree $d$ is 
increased. This situation holds in particular when $f$ is evaluated by a substantial applications code. Moreover, the approximation of the adjoint vector $a$ by differencing would be quite costly, as it involves at least $n$ evaluations of $f$. For this reason many authors restrict the evaluation of such gradients to subspaces through the use of Brown/Brent-like modifications [3] of Newton's method. By automatic differentiation any vector of the form $a$ can be obtained at a cost that is independent of $n$ relative to the cost of evaluating $f$ itself.

\section{Directional Derivatives and Mixed Partials}

Whenever the $d$ vectors $v_{i}$ in (1) are identical to a fixed vector $v \in \mathrm{R}^{n}$, we will call $b$ the directional derivative of $f$ along $v$. Dividing this $b$ by $d$ !, we obtain a Taylor coefficient denoted by $y^{(d)}(v)$, so that for fixed $x$

$$
y(t)=f(x+t v)=\underbrace{y^{(0)}+t \cdot y^{(1)}(v)+t^{2} \cdot y^{(2)}(v)+\ldots+t^{d} \cdot y^{(d)}(v)}_{f_{d}(t \cdot v) \equiv}+\mathcal{O}\left(t^{d+1}\right) .
$$

Here we have assumed that $f$ is at least $d$ times Lipschitz-continuously differentiable. We introduced this truncated Taylor series because its coefficients $y^{(j)}(v)$ can be evaluated directly much more easily than the more general mixed derivatives $b$ given in (1). Moreover the latter can be obtained from the univariate Taylor coefficients by the following Lagrangian interpolation technique. Abbreviating $w \equiv t \cdot v$, we find that the function $f_{d}(w)$ defined in (3) is a multivariate polynomial of degree $d$ in $w \in \mathbf{R}^{n}$. The jet $f_{d}$ represents exactly the values of the up to $d$-th derivatives of $f$ at the fixed base point $x$. Provided $d$ is sufficiently large, we can expect that the solution set of the algebraic bifurcation equations $f_{d}(w)=0$ is locally homeomorphic to that of the original system $f(x)=0$. Fortunately, it is usually sufficient to analyze $f_{d}(w)$ on a subspace spanned by the $\hat{n}<<n$ columns of a matrix $V$. While we consider the individual columns of $V$ as constant, their number-like the degree $d$-may be successively enlarged.

Now suppose we wish to obtain the coefficients or some other computationally convenient representation of the polynomial

$$
\phi_{d}(z) \equiv f_{d}(V z)
$$

Being a $d$-th degree polynomial in $\hat{n}$ variables, $\phi_{d}$ has $\hat{n}+d$ choose $d+1$ unknown coefficients. To tie down these degrees of freedom, one needs at least the same number of values $\phi_{d}(g)=f_{d}(\mathrm{Vg})$ at suitable sample points $g \in \mathbf{R}^{\hat{n}}$. To make do with that minimal number and to obtain a convenient Lagrangian interpolation formula, we may let $g$ range over all integer vectors whose $\hat{n}$ components $g_{j}$ are nonnegative and sum to $d$ or less. There is an obvious 1-1 correspondence between these lattice vectors and the homogeneous terms with multiindex $g$ in the expansion of $\phi_{d}(z)$. Moreover, with $e$ the $\hat{n}$-vector of ones and $g$ any of the lattice points one can easily check that the polynomial

$$
L_{g}(z)=\prod_{i>e^{T} z}^{i \leq d}\left(i-e^{T} z\right) \cdot \prod_{i=0}^{i<g_{1}}\left(z_{1}-i\right) \cdots \prod_{i=0}^{i<g_{j}}\left(z_{j}-i\right) \cdots \prod_{i=0}^{i<g_{\dot{n}}}\left(z_{\hat{n}}-i\right)
$$


has degree $d$ and vanishes at all grid points except $g$ itself. Thus we obtain for any argument $z$ the vector polynomial identity

$$
\phi_{d}(z)=\frac{1}{d !} \sum_{0 \leq g}^{e^{T} g \leq d}\left(\begin{array}{c}
e^{T} g \\
g_{1} \ldots g_{\tilde{n}}
\end{array}\right) \cdot \phi_{d}(g) \cdot L_{g}(z) r,
$$

where the first term under the sum is the multinomial coefficient representing $d ! / L_{g}(g)$.

When $d$ and $\hat{n}$ are small integers, this Lagrangian formula provides easy access to the values and derivatives of $f_{d}$ at arbitrary arguments. It should also be noted that an additional column in $V$ and thus an additional component in $z$ can be accommodated without resampling on the previous subspace. Similarly, one can reuse the old sample values after an increase in the degree $d$. By differentiating (6) with respect to the vector $z$ and then evaluating at the origin $z=0$, all partials of $f$ with respect to the columns of $V$ can be expressed as linear combinations of the sample values $\phi_{d}(g)$. Consequently, adjoints of the mixed partials can also be obtained as adjoints of the vectors $\phi_{d}(g)$ considered as a function of $x$. Hence we conclude that the problem of calculating mixed partials and their adjoints can be reduced to that of calculating directional derivatives (or more precisely univariate Taylor coefficients) and their adjoints.

It is also possible to directly calculate multivariate Taylor series of $f$ on the range of some $V$ by automatic differentiation. As of now, we prefer instead the repeated evaluation of univariate Taylor coefficients because it allows for much simpler and smaller data and program structures. Also, if the subspace needs to be enlarged, it would be impossible to update the mu!tivariate Taylor series unless an enormous amount of intermediate data has been stored. This assertion will become clearer in the following section.

\section{Propa $_{\circ}$ ation of Taylor Series and Their Adjoints}

Virtually all vector functions $y=f(x): \mathbf{R}^{n} \rightarrow \mathbf{R}^{m}$ of practical interest are evaluated by computer prograrns whose execution consists of a sequence of scalar assignments, that is, a loop of the following form:

\section{Original Program}

$$
\begin{gathered}
\text { For } i=n+1, n+2, \ldots, n+p+m \\
s_{i}=f_{i}\left\langle s_{j}\right\rangle_{j \in \mathcal{J}_{i}}
\end{gathered}
$$


Here each elementary function $f_{i}$ depends on already-computed scalar quantities $s_{j}$, with $j$ belonging to an index set

$$
\mathcal{J}_{i} \subset\{1,2, \ldots, i-1\} \quad \text { for } \quad i=n+1, n+2, \ldots, n+p+m
$$

The nonnegative integer $p$ represents the number of intermediate variables, which we expect to be much larger than both $n$ and $m$ for seriously nonlinear problems. Thus we can combine the variables $s_{i}$ into three vectors:

$$
\begin{aligned}
& x \equiv\left(s_{1}, s_{2}, \ldots \quad \ldots, s_{n-1}, s_{n}\right) \quad \text { (independent) } \\
& z \equiv\left(s_{n+1}, \ldots \quad \ldots, s_{n+p}\right) \quad \text { (intermediate) } \\
& y \equiv\left(s_{n+p+1}, \ldots \quad \ldots, s_{n+p+m}\right) \quad \text { (dependent) }
\end{aligned}
$$

On a parallel machine, components of $z$ and $y$ that do not directly or indirectly depend on each other can be evaluated concurrently. On a sequential machine, the cost of executing all assignments is largely independent of the variable ordering.

In mathematical terms $f$ is the composition of $p+m$ elementary or library functions $f_{i}$, which are assumed to be analytic in the interior of their domains For example, this is clearly the case when all $f_{i}$ represent either elementary arithmetic operations ( namely,,,$+- *$, and / ) or nonlinear system functions of a single argument ( for example, logarithms, exponentials, and trigonometric functions).

Rather than restricting ourselves to unary and binary elementary functions, we may allow any number of arguments. However, we do need the property that, given a truncated Taylor series

$$
s_{j}(t)=s_{j}^{(0)}+s_{j}^{(1)} t+s_{j}^{(2)} t^{2}+\ldots+s_{d}^{(d)} t^{d}+\mathcal{O}\left(t^{d+1}\right)
$$

for all $j \in \mathcal{J}_{i}$, the corresponding coefficients of $s_{i}(t)=f_{i}\left(s_{j}(t)\right)_{j \in \mathcal{J}_{i}}$ can be computed at an $\mathcal{O}\left(d^{2}\right)$ cost. Here the computational cost may be measured in any reasonable way accounting in particular also for reads and writes from and to memory. Using the sum, product, and quotient rule of differentiation, one can easily check that this condition is met for all arithmetic operations. Similarly, one may use the fact that all standard functions are quadratures or solutions to linear ODEs in order to show that Taylor series can be propagated from their arguments to their values with quadratic complexity in $d$. In the Appendix we list the required calculations for the natural logarithm and its adjoint in detail. Obviously, the same complexity requirement holds also for multivariate linear and diagonally quadratic functions.

Combining the first $(d+1)$ Taylor coefficients of $s_{i}$ into a vector

$$
S_{i} \equiv\left(s_{i}^{(0)}, s_{i}^{(1)}, s_{i}^{(2)}, \ldots, s_{i}^{(d)}\right)^{T},
$$

we may thus expand the original program to a recursion on Taylor series: 


\section{Forward Sweep}

$$
\begin{gathered}
\text { For } i=n+1, n+2, \ldots, n+p+m \\
S_{i}=F_{i}\left\langle S_{j}\right\rangle_{j \in \mathcal{J}_{i}}
\end{gathered}
$$

where the vector functions $F_{\mathbf{i}}: \mathbf{R}^{d+1} \rightarrow \mathbf{R}^{d+1}$ are uniquely determined by the underlying scalar functions $f_{i}$. The transition from $f_{i}$ to $F_{i}$ can be interpreted as an extension of real or complex arithmetic to an algebra of truncated Taylor series ([13]). In any case we can ensure that for some constant $c>0$ the evaluation costs of $f_{i}$ and $F_{i}$ satisfy the simple relation.

$$
\operatorname{Cost}\left\{F_{i}\left\langle S_{j}\right\rangle_{j \in \mathcal{J}_{i}}\right\} / \operatorname{Cost}\left\{f_{i}\left\langle s_{j}\right\rangle_{j \in \mathcal{J}_{i}}\right\} \leq c \cdot(1+d)^{2} .
$$

Now let us consider the composite vector function $F$ that maps a truncated Taylor series for a path $x(t)$ into the corresponding approximation to $y(t)=F(x(t))$. Since this function can be evaluated by one forward sweep, it follows from the last inequality by summation over $i$ that at (least on a serial machine)

$$
\operatorname{Cost}\{F(X)\} / \operatorname{Cost}\{f(x)\} \leq c \cdot(1+d)^{2} .
$$

Here $X=\left[x_{i}^{(j)}\right]$ may be thought of as a matrix with $n$ rows $X_{i}$ and $(d+1)$ columns $x^{(j)}$. Similarly $Y=\left[y_{i}^{(j)}\right]$ forms a matrix with $m$ rows $Y_{i}$ and $(d+1)$ columns $y^{(j)}$.

To recover from these Taylor series the derivative vectors discussed in Section 2, we may restrict $x(t)$ to be linear, so that only the first two columns $x \equiv x^{(0)}$ and $v \equiv x^{(1)}$ of $X$ are nonzero. Then the resulting $(d+1)$ columns of $Y=F(X)$ are exactly the coefficient vectors occurring in equation (3). The software package ADOL-C briefly described in the following section contains a problemindependent routine forward for calculating $Y$ given $x$ and $v$, and after the structure of the composite function $f$ has been recorded during an execution of the original program.

Finally, let us consider the problem of computing for fixed $x, v$, and some $u \in \mathbf{R}^{m}$ the adjoint values

$$
\bar{s}_{i}^{(j)} \equiv \frac{\partial\left\langle u^{T} y^{(d)}\right\rangle}{\partial s_{i}^{(j)}} .
$$

The adjoints $\bar{s}_{i}^{(j)}$ describe the sensitivity of a weighted sum of the highest Taylor coefficients $y_{i}^{(d)}$ with respect to the intermediate coefficients $s_{i}^{(j)}$. In particular, we find for $j=0$ and $i \leq n$ that

$$
\ddot{x}_{i} \equiv \bar{s}_{i}^{(0)}=\frac{\partial}{\partial x_{i}} u^{T} \nabla^{d} f(x) v^{d}
$$

is the $i$-th component of the vector $a$ defined in (2) with all $v_{i}$ identical. It can also be seen that the other columns $\bar{x}^{(j)}$ of the matrix $\bar{X}$ represent the vectors a defined in (2) with $d$ replaced by $d-j$. The salient fact about the scalar coefficients $\bar{s}_{i}^{(j)}$ is that, like the underlyirir, $s_{i}^{(i)}$ they can 
be computed recursively, with the original order of dependency exactly reversed. More specifically, $\bar{s}_{i}^{(j)}$ may depend on all $\bar{s}_{i}^{(\bar{j})}$ with $\tilde{i} \geq i$ and $\tilde{j} \geq j$. It follows from the chain rule that each vector $\bar{S}_{j}$ can be computed from the vectors $\bar{S}_{i}$ with $j \in \mathcal{J}_{i}$ by using the Jacobians $\nabla F_{i}$ of the corresponding mappings $F_{i}$ at the given point. After initializing all $\bar{S}_{i}$ except the components of $\bar{Y}$ to zero, one must execute the following loop:

\section{Reverse Sweep}

$$
\text { For } \begin{aligned}
i= & n+p+m, n+p+m-1, \ldots, n+1 \\
& \bar{S}_{j}^{T}+=\bar{S}_{i}^{T}\left[\frac{\partial}{\partial S_{j}} F_{i}\right] \quad \text { for } \text { all } j \in \mathcal{J}_{i}
\end{aligned}
$$

where $a+=\dot{b}$ means increment $a$ by $b$ as in the programming language C. Since the mappings $F_{i}$ arise from single elementary operations and functions, they are highly structured, and one can perform the adjoint evaluations without actually forming the Jacobian matrix of the $F_{i}$. In fact, the adjoint evaluations associated with any $F_{i}$ can usually be carried out at no more than twice the computational effort of evaluating $F_{i}$ itself (see the Appendix). However, one does have to store the current arguments $S_{j}$ of each $F_{i}$ during the forward sweep in order to access this information on the reverse sweep. The size of this data set is proportional to the original run time and may therefore grow extremely large. Fortunately, since the data are accessed strictly sequentially, they can be stored and retrieved from disk without severe run-time penalties. The amount of data that need to be accessed at random can be limited to $2(d+1)$ times the core memory requirement of the original program. The routine reverse described below calculates $\bar{X}$, given $u$ and implicitly $x, v$.

If multivariate rather than univariate Taylor series are propagated during the forward sweep, the amount of information that has to be stored in preparation for the reverse sweep grows significantly. The same amount of storage is needed if one does not plan a reverse sweep but merely wishes to increase the degree $d$ or the dimension $\hat{n}$ of the subspace recursively, without regenerating terms that were already calculated during earlier sweeps. If the final $\hat{n}$ and $d$ are known a priori and are of significant size, it might be efficient to implement the functions $F_{i}$ with fast methods [2], for example, using Fourier transforms. This sophisticated approach would reduce the complexity per elementary operation to an order of $d \log d$ rather than the order $d^{2}$ of the naive method based on explicit polynomial multiplication. Fortunately, the quadratic term is initially small compared to the affine part and the general overhead, so that the run-time growth appears to be essentially linear for $d<9$. 


\section{Implementation by Overloading}

The mathematical techniques sketched above have been implemented in various ways. Apart from computational efficiency and accuracy, user convenience must be a leading design objective. The first question is how the user specifies the nonlinear system $f(x)=0$. Sometimes $f(x)$ may have a concise algebraic representation; sometimes it may be generated automatically, for example, by a finite element package. In large-srale problems it seems likely that the vector function $f(x)$ will at some stage be represented by an evaluation code in a high-level programming language like Fortran, Algol, or C. Assuming that this is the case, such given code is the natural object for automatic differentiation. Early on, some researchers advocated and practiced the modification of the original code by hand (for a survey, see [11]). While this hand coding is obviously rather tedious and error prone for problems of any size, an experienced programmer can probably write an adjoint code that is smaller and more efficient than automated alternatives. In fact, certain features (e.g., implicit functions and other subprograms) are handled inefficiently or not at all by the automatic differentiation packages that we are aware of.

Several researchers developed precompilers that analyze a given code (usually Fortran 77) and add the extra instructions that are needed to compute the required derivatives (see, e.g., [8], and [9]). The resulting expanded code is then compiled with a standard compiler into an object code that can simultaneously evaluate derivatives if called with appropriate options. A more direct route to the same result can be taken in languages that allow the overloading of operators and functions for arguments of user-defined types. With the help of problem-independent header files, one may then coax the compiler itself to issue the required extra instructions. Essent: "ly, all the user has to do is to redeclare his floating-point variables to be of a different type (see, e.g., [14]). Unfortunately, the overloading capability is currently not available in Fortran, but it will be included in Fortran 90. Using the public-domain converter ${ }^{2} \mathbf{c}$ of AT\&T, one can translate codes written in Fortran 77 $\mathrm{C}$, which is essentially a subset of $\mathrm{C}++$ where overloading is possible. The routines for performing subsequent reverse and forward sweeps may then again be called from a Fortran program (e.g., an optimization package). We have traveled this circuitous route for a weather model based on the shallow water equation, as reported in the following section. For the automatic differentiation of the $\mathrm{C}$ code, we used our $\mathrm{C}++$ package ADOL- $\mathrm{C}$, which is documented in [6] and can be obtained free of charge from the author.

To convey an idea of how ADOL-C is used, let us consider the following univariate example : 


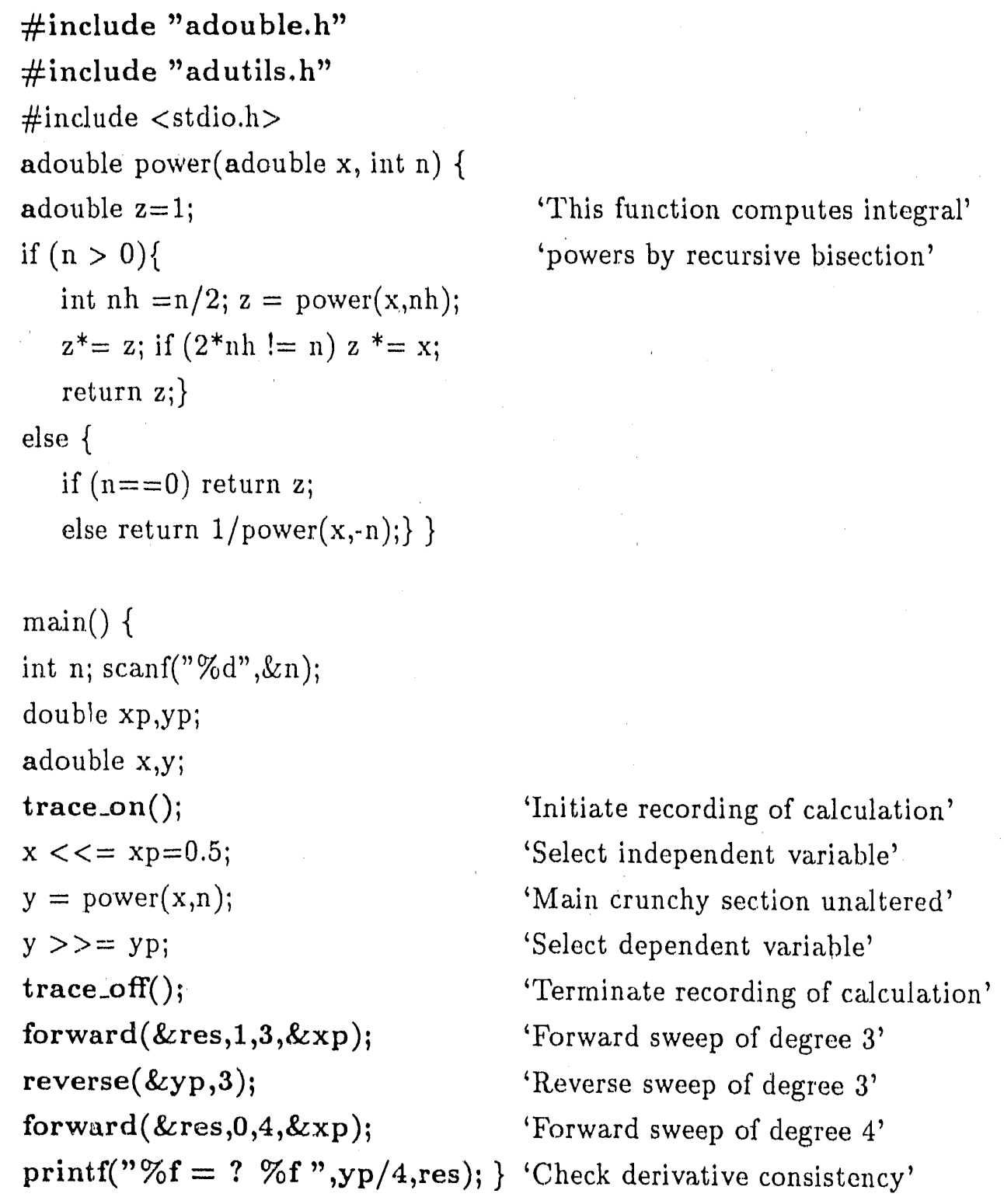

The letters and words printed in boldface had to be added in order to make the original program fit for differentiation. The quoted header files contain the definition of the new type adouble to which all floating-point variables that are considered as differentiable functions must be redeclared. Independent and dependent variables are selected with the special assignment operators $<<=$ and $>>=$. All calculations between the function calls trace_on and trace_off are internally recorded on a tape. Subsequrntly this tape can be used to calculate direct and adjoint derivatives of arbitrary order. In this univariate case the reverse sweep has no advantage, but its results for some $d$ can be compared to those from the forward sweep with $d$ incremented by one. The example shows that even recursive subprogram calls are no problem. Similarly, one may have branches as in the function power, provided they are not conditioned on the values of adoubles. 
On problems with many independent and dependent variables, the modifications of the original $\mathrm{C}$ code are exactly the same, except that there must of course be several special assignments of the furm $<<=$ and $>>=$. Also, the derivative evaluation routines forward and reverse need more arguments; that is, their calls must be of the form

$\begin{array}{ll}\text { void forward }(\mathrm{Y}, \mathrm{rev}, \mathrm{deg}, \mathrm{x}, \mathrm{v}) \\ \text { double } \mathrm{Y}[\mathrm{m}][] ; & / / \text { columns as in }(1) \text { for } \mathrm{d} \leq \mathrm{deg} \\ \text { int rev ; } & / / \text { flag for subsequent reverse } \\ \text { int deg ; } & / / \text { maximal degree of derivatives } \\ \text { double } \mathrm{x}[] ; & / / \text { independent variable vector } \\ \text { double } \mathrm{v}[] ; & / / \text { tangent vector in domain }\end{array}$

void reverse(Xbar,deg,u)

double Xbar[n][] ; $\quad / /$ columns as in (2) for $d \leq \mathrm{deg}$

int deg;

$/ /$ maximal degree of derivative -1

double $u[] ; \quad / /$ adjoint weight vector

where $m$ and $n$ are the (internally stored) numbers of dependent and independent variables, respectively. A call to reverse must always be preceded by a corresponding call to forward with rev=1. As we have stressed before, the run time for either routine is bounded by a constant multiple of $(1+d)^{2}$ relative to the run time of the underlying undifferentiated evaluation program.

The package includes convenient drivers for the evaluation of gradients and Hessians. A driver that calculates full-derivative tensors on subspaces with the Lagrangian formula (6) is currently under development.

\section{Numerical Results}

To verify the practical possibility of calculating higher-order derivatives for sizable problems, we consider the classical Bratu problem and a shaliow water model for weather modeling [12].

For the Bratu problem we chose a cylindrical geometry with homogeneous Dirichlet boundary conditions and an Arrhenius term with two parameters. These were considered as independent variables together with the temperature distribution. Rather than actually calculating the cubic turning point of this system, we simply calculated direct and adjoint derivative vectors of degree 0 to 8 at an arbitrary point. Under the assumption of rotational symmetry, the problem is reduced to a rectangle and then discretized by using bilinear elements on an orthogonal but unevenly spaced 
grid. The original Fortran code was hand translated into $\mathrm{C}$ and then modified for automatic differentiation as indicated in the previous section. The results are shown in the following table.

\begin{tabular}{|c|c|c|c|c|c|c|c|c|}
\hline Grid & \multicolumn{2}{|c|}{$10 \times 20$} & \multicolumn{2}{|c|}{$20 \times 40$} & \multicolumn{2}{|c|}{$30 \times 60$} & \multicolumn{2}{|c|}{$40 \times 80$} \\
\hline Tape & \multicolumn{2}{|c|}{59392} & \multicolumn{2}{|c|}{272384} & \multicolumn{2}{|c|}{641024} & \multicolumn{2}{|c|}{1163264} \\
\hline Deaths & \multicolumn{2}{|c|}{7081} & \multicolumn{2}{|c|}{32441} & \multicolumn{2}{|c|}{76201} & \multicolumn{2}{|c|}{138361} \\
\hline Original & \multicolumn{2}{|c|}{0.085} & \multicolumn{2}{|c|}{0.41} & \multicolumn{2}{|c|}{1.0} & \multicolumn{2}{|c|}{1.7} \\
\hline Mode & For. & Rev. & For. & Rev. & For. & Rev. & For. & Rev. \\
\hline $\mathrm{d}=0$ & 5.6 & 8.6 & 4.8 & 8.3 & 4.9 & 8.4 & 4.9 & 8.4 \\
\hline $\mathrm{d}=2$ & 12.6 & 24.8 & 12.4 & 23 & 11.6 & 22.8 & 12.4 & 23.8 \\
\hline $\mathrm{d}=4$ & 24.6 & 49.4 & 23.1 & 45.2 & 22.2 & 45.2 & 23.2 & 45.2 \\
\hline $\mathrm{d}=6$ & 40.8 & 82.4 & 37.8 & 75 & 36.6 & 73.6 & 37.6 & 77.2 \\
\hline $\mathrm{d}=8$ & 61 & 121.4 & 55.4 & 111.9 & 55.5 & 113.4 & 55.9 & 111.6 \\
\hline
\end{tabular}

The first two rows, called tape and deaths, give the sequential storage requirement for forward and reverse sweeps, respectively, as tape $+8(d+1)$ deaths bytes and tape $+16(d+1)$ deaths bytes. The factor 2 in the storage requirement for reverse can be avoided if adjoint derivative vectors are computed only in single-precision accuracy. As always, the amount of randomly accessed core memory is roughly $(d+2)$ times that of the original code, which is in this case negligible. The entries in the third row represent the run time of the undifferentiated code on a Sun 3 (in seconds). All entries underneath are ratios between the run time of forward or reverse sweeps and the corresponding times in the third row. As one can see, the reverse sweeps take some $50-100 \%$ longer than forward sweeps, and the times for both grow roughly linearly in $(d+1)$.

A similar picture emerges for the second example, the discretization of three coupled hyperbolic PDEs with two spatial dimensions. Here we considered only one $11 \times 11 \times 30$ grid. The 3,483 independent variables represent the initial and boundary conditions on all but the terminal face of the box. Starting from the initial face, the shallow-water equation is integrated over 30 time steps. The ostensible purpose of the calculation is to minimize a weighted sum of errors between calculated and observed values at the 3,630 grid points by varying the independent variables representing the initial conditions. For this purpose the original Fortran code contained a separate subroutine for the generation of artificial data in common blocks. Only the routine for the subsequent evaluation of the sum of squares residual was translated into $\mathrm{C}$ by using the converter f2c of AT\&:T. After the minor modifications discussed above, the $\mathrm{C}++$ code was compiled and then linked with the object codes generated from the Fortran parts of the program. Our utilities forward and reverse were also called from Fortran, which works because their parameters were deliberately restricted to compatible data types. The results of the calculation are again listed as run-time ratios relative to the five seconds taken by the execution of the original code.

\begin{tabular}{|c|c|c|c|c|c|c|}
\hline $\mathrm{d}$ & 0 & 1 & 2 & 4 & 6 & 8 \\
\hline Forward & 3.357 & 5.046 & 6.914 & 11.36 & 18.77 & 25.82 \\
\hline Reverse & 5.691 & 8.874 & 12.411 & 20.93 & 34.88 & 48.55 \\
\hline
\end{tabular}


The total storage I ?quirement for these runs varied between 2.5 and 25 micgabytes. Again we see that the run time grows essentially linearly in the degree $d \leq 8$, with the reverse sweeps taking almost twice as long as the corresponding forward sweeps. Overall we conclude that direct and adjoint derivative actors can be computed at a reasonable cost in terms of run time. The extra storage requirement for the reverse sweeps is substantial but manageable. The reader may wonder why we did not discuss the accuracy of the numerical derivative obtained. The reason is that, on all test problems for which handcoded derivatives are available, their numerical values are virtually identical with those obtained by ADOL-C. It should be noticed that automatic differentiation does not incur any truncation errors and would yield exact results if the calculations could be carried out with infinite precision. Further investigations and software development should target the automatir difierentiation of implicit functions and numerical approximations to Poincaré maps.

\section{Appendix}

As a typical example of a univariate system function we examine the natural logarithm as an $\epsilon^{\prime}$ omentary function, namely, $s_{i}=f_{i}\left(s_{j}\right) \equiv \log \left(s_{i}\right)$. Assuming that the scalars $s_{j}$ and consequently $s_{i}$ are in fact smootb functions of a parameter $t \in \mathbf{R}$, we find by differentiation that for all $k \geq 1$

$$
\left[s_{j}(t) s_{i}(t)^{\prime}\right]^{(k-1)} \equiv s_{j}(t)^{(k)}
$$

where $s(t)^{(k)}$ denotes the $k$-th Taylor coefficient of $s(t)$ with respect to $t$. By identifying terms on both sides, one finds the recurrence

$$
\tilde{s}_{i}^{(k)}=\frac{1}{s_{j}}\left[s_{j}^{(k)}-\sum_{l=1}^{k-1} \tilde{s}_{i}^{(l)} s_{j}^{(k-l)}\right] \quad \text { and } \quad s_{i}^{(l)}=\frac{1}{l} \tilde{s}_{i}^{(l)} \quad \text { for } 1 \leq l \leq d \text {. }
$$

This simple algorithm evaluates the vector function $S_{i}=F_{i}\left(S_{j}\right)$ for $f_{i}=\log$ by using $d$ divisions and $d(1+d) / 2$ multiplications followed by additions or subtractions. The corresponding adjoint operation can be effected by the recurrences $\overline{\tilde{s}}_{i}^{(k)}=\frac{1}{k} \bar{s}_{i}^{(k)}$ for $1 \leq k \leq d$ and

$$
\begin{aligned}
& \overline{\tilde{s}}_{i}^{(l)}-=\frac{1}{s_{j}}\left[\sum_{l=i+1}^{d} \overline{\bar{s}}_{i}^{(k)} s_{i}^{(k-l)}\right], \text { for } l=d-1, \ldots, 1 \\
& \tilde{s}_{j}{ }^{(l)}+=\frac{1}{s_{j}}\left[{\overline{\bar{s}_{i}}}^{(l)}-\sum_{k=l+1}^{d}{\overline{\bar{s}_{i}}}^{(k)}{\tilde{s_{i}}}^{(k-l)}\right], \quad \text { for } \quad 0 \leq l \leq d
\end{aligned}
$$

When $d$ is of some size, these adjoint recurrerces involve almost exactly twice as much work as the original ones for the evaluation of $F_{i}$. The logarithm is in this respect typical of the other transcendenta! functions and the multiplication operation. As a consequence, reverse sweeps tend to take almost twice as long as the corresponding forward sweeps, as was certainly true for our numerical resu'.'s. 


\section{Acknowledgments}

The author is indebted to B. Fiedler and P. Kunkel for advice, to I. M. Navon for providing the shallow water code, and to S. Reese for conducting the numerical experiments. The work on this project was began during a three week visit at the Konrad Zuse Zentrum in Berlin.

\section{References}

[1] W.J. Beyn (1991). Numerical Methods for Dynamical Systems, to appear in the Proceedings of the SERC Summer School in Lancaster, England.

[2] R. P. Brent and H. T. Kung (1978). Fast Algorithms for Manipulating Power Series, J. of the ACM, Vol. 25, pp. 581-595.

[3] K. Brown (1969). A Quadratically Convergent Method for Solving Nonlinear Equations, SIAM J. Numer. Analysis, Vol. 6, pp. 560-569.

[4] A. Griewank (1989). "On Automatic Differentiation," in Mathematical Programming: Recent Developments and Applications, ed. M. Iri and K. Tanabe, Kluwer Academic Publishers, pp. 83-108.

[5] A. Griewank (1990). Direct Calculation of Newton Steps without Accumulating Jacobiars, Preprint MCS-P127-0290, Argonne National Laboratory, Argonne, Ill. To appear in the Proceedings of the SIAM Workshop on Large-Scale Optimization, held at Cornell University in 1989.

[6] A. Griewank, D. Juedes, and J. Srinivasan (1990). ADOL-C, A Package for the Automatic Differentiation of Algorithms Written in $C / C++$, Preprint MCS-180-1190, Argonne National Laboratory, Argonne, 11 .

[7] A. Griewank and G. W. Reddien (1989). Computation of Cusp Singularities for Operator Equations and Their Discretizations, J. Comp. and App. Math., Vol. 48, pp. 591-601.

[8] J. E. Horwedel, B. A. Worley, F. M. Oblow, and F. G. Pin (1988). GRESS Version 0.0 Users Manual, ORNL/TM 10835, Oak Ridge National Laboratory, Oak Ridge, Tenn.

[9] K. Kubota and M. Iri (1990). Padre 2, version 1-User's Manual, Research Memorandum RMI 90-01, Faculty of Engineering, University of Tokyo, Hongo 7-3-1, Bunkyo-ku, Tokyo.

[10] P. Kunkel (1988). Quadratically convergent methods for the computation of unfolded singularities SIAM J.Numer. ANAL. Vol. 25, pp. 1392-1408. 
[11] H. Kagiwada, R. Kalaba, N. Rosakhoo, and K. Spingarn (1986). Numerical Derivatives and Nonlinear Analysis. In Mathematical Concepts and Methods in Science and Engineering, Vol. 31, ed. A. Miele, Plenum Press, New York.

[12] I. M. Navon and U. Muller (1979). FESW-A Finite-Element Fortran IV Program for Solving the Shallow Water Equations, Advances in Engineering Software, Vol. 1, pp. 77-84.

[13] L.B. Rall (1990). Differentiation Arithmetics. In Computer Arithmetic and Self-validating Numerical Methoids, Notes and Reports in Mathematics in Science and Engineering, Vol. 7, pp. 73-90, Academic Press, Boston.

[14] L. B. Rall (1984). Differentiation in PASCAL-SC: Type GRADIENT, ACM TOMS, Vol. 10, pp. $161-184$.

\section{DISCLAIMER}

\footnotetext{
This report was prepared as an account of work sponsored by an agency of the United States Government. Neither the United States Government nor any agency thereof, nor any of their employees, makes any warranty, express or implied, or assumes any legal liability or responsibility for the accuracy, completeness, or usefulness of any information, apparatus, product, or process disclosed, or represents that its use would not infringe privately owned rights. Reference herein to any specific commercial product, process, or service by trade name, trademark, manufacturer, or otherwise does not necessarily constitute or imply its endorsement, recommendation, or favoring by the United States Government or any agency thereof. The views and upinions of authors expressed herein do not necessarily state or reflect thuse of the United States Government or any agency thereof.
} 

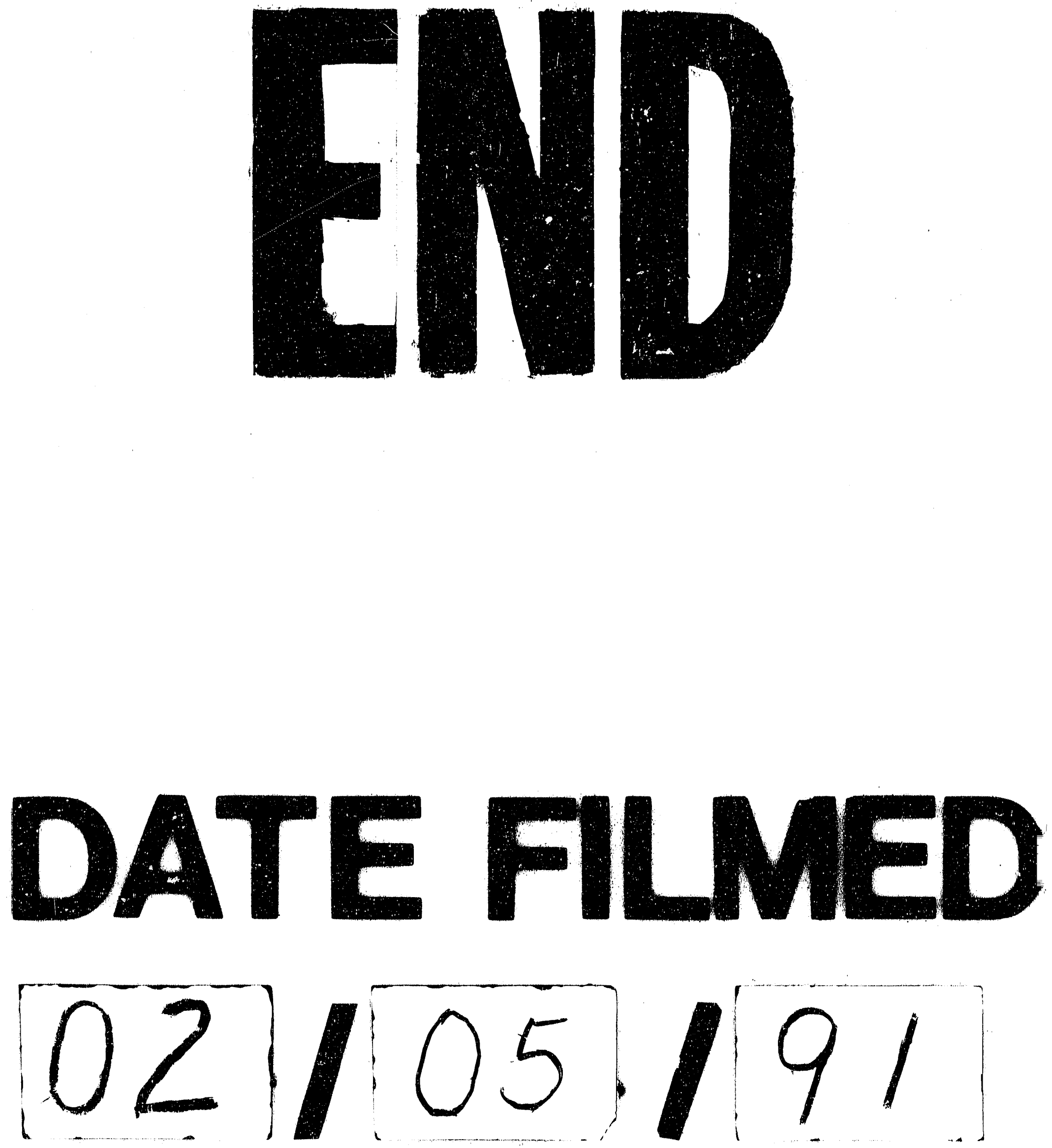
